Egyptian Journal of Chemistry

http://ejchem.journals.ekb.eg/

\title{
Synthesis of New Bioactive Compounds of Pyrazino [2,3-e][1,3] Oxazepines and Pyrazino [2,3-e] [1,3] Diazepines Bearing 1,3,4-Oxadiazole Moieties
}

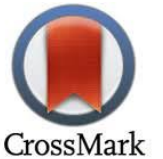

\author{
A. N. Ayyash" ${ }^{* 1}$, K. A. Juwair ${ }^{2}$, O. G. Najeeb ${ }^{1,2}$, N. M. Jasim ${ }^{1}$ \\ ${ }^{1}$ University of Fallujah, College of Applied Science, Department of Applied Chemistry, Anbar, Iraq \\ ${ }^{2}$ University of Fallujah, Biotechnology and Environmental Center, Anbar, Iraq
}

\begin{abstract}
A series of new derivatives of 7,7'-(1,4-phenylene)bis[(5-substitutedphenyl-1,3,4-oxadiazol-2-yl)-amino(7,8dihydropyrazino[2,3-e][1,3]oxazepine-5,9-dione)] 3a-d and 7,7'-(1,4-phenylene)bis[(5-substitutedphenyl-1,3,4-oxadiazol-2yl)-amino(7,8-dihydro-5H-pyrazino[2,3-e][1,3]diazepine-5,9(6H)-dione)] $4 \mathrm{a}$-d have been prepared by the cycloaddition reaction of 2,3-pyrazinedicarboxylic anhydride (and/or 2,3-pyrazinedicarboximide) with new derivatives of 1,3,4-oxadiazole bearing azomethine group which were prepared via cyclization reaction of some carbohydrazides with various benzoic acids in $\mathrm{POCl}_{3}$. The structures of the desired compounds were in a coincidence as deduced from their FTIR, ${ }^{1} \mathrm{H}$ NMR, ${ }^{13} \mathrm{C}$ NMR, and mass spectra. Antimicrobial activities of titled compounds have been recorded with good to excellent results.
\end{abstract}

Keywords: Diazpines; Oxazepines; 1,3,4-Oxadiazole; Carbohydrazide; Pyrazinedicarboxylic.

\section{Introduction}

Five and seven membered heteroaromatic systems with two or more heteroatoms are an important class of heterocyclic and bioactive compounds. Diazepine and oxazepine compounds bearing oxadiazole rings represent a good example and classified as antidepressant, antibacterial and antifungal drugs [15]. So, synthesis and development of new diazepines and oxazepines has attracted remarkable frameworks. However, according to the literature review and in the last five years, a large number of oxadiazole, diazepine and oxazepine derivatives have been prepared by various synthetic procedures. For example, some derivatives of 1,3,4-oxadiazole were prepared via reaction of benzohydrazides with substituted benzoic acid in the presence of $\mathrm{POCl}_{3}[6]$. Fused 1,3,4-oxadiazole derivatives were achieved by the dehydration of dioximes with acetic anhydride [7]. In contrast, new derivatives of benzodiazepine4,7-dione were synthesized by cycloaddition reaction of heteroaromatic Schiff bases and phthalimide under refluxing in dry THF as a solvent [8]. Whereas, the cyclocondensation of 1,3-diketone compounds with 2-aminoethanethiols in pyridine gave 1,4-thiazepine and 1,5-thiazepine derivatives [9]. Further, 1,3-
oxazepin-5(1H)-one derivatives have been obtained from the reaction of imines and isobenzofuranone [10]. Also, another oxazepineones derivatives were prepared by the treatment of aminomethylisoxazole with salicylaldehydes followed by reduction with $\mathrm{NaBH}_{4}[11]$.

So, and as our interesting, it was necessary to contemplate about designing and synthesis a new derivatives of bis-pyrazino[2,3-e][1,3] and pyrazino[2,3-e][1,3] diazepines as a novel heteroaromatic compounds bearing 1,3,4-oxadiazole ring and investigation of their antifungal activity.

\section{Experimental}

All chemicals were used as received from their suppliers companies without more purification. For monitoring reactions, TLC technique was applied with pre-coated aluminum plates. Determination of melting points were recorded with open capillary tubes and are uncorrected. Infra-red spectra (FTIR) were outlined on 8400s FTIR-Shimadzu spectrophotometer using $\mathrm{KBr}$ disc and expressed as a wave number $\left(\mathrm{cm}^{-1}\right)$. The ${ }^{1} \mathrm{H}$ and ${ }^{13} \mathrm{C}$ NMR spectra were done on Bruker spectrometer 400 and $100 \mathrm{MHz}$, respectively, in DMSO- $\mathrm{d}_{6}$ solvent and (TMS) as internal standard reference. Microanalysis of

*Corresponding author e-mail: Ahmedn.ayyash@uofallujah.edu.iq

Receive Date: 29 December 2020, Revise Date: 24 January 2021, Accept Date: 14 March 2021

DOI: 10.21608/EJCHEM.2021.55735.3174

(C2021 National Information and Documentation Center (NIDOC) 
elements $(\mathrm{C}, \mathrm{H}$, and $\mathrm{N})$ data were founded by the Elemental Analyzer Model Fison (EA1108). GCMS QP-1000 EX MS technique was utilized for mass spectra. All the chemical structures were predicted with the ChemDraw Ultra (6.0) software application.

\section{Synthesis of $\quad N^{\prime}, N^{\prime \prime}-[1,4-$ phenylenedimethanylylidene]bis(2 -aminoaceto hydrazide), compound 1:}

1,4-phenyldialdehyde (terephthaldehyde) (0.01 mol), carbohydrazide $(0.02 \mathrm{~mol})$, and glacial acetic acid (3 drops) were dissolved in absolute ethanol $(30 \mathrm{~mL})$ and heated to reflux for 2 hours. The solid precipitated was recrystallized from ethanol [12].

Synthesis of 2,2'-\{1,4phenylenebis[methanylylidene hydrazin-1-yl-2ylidene]\}bis[5-(4-substitutedphenyl)-1,3,4oxadiazole], compounds 2a-d:

Compound 1 (2.78g, $0.01 \mathrm{~mol})$, 4-substituted benzoic acid $(0.02 \mathrm{~mol})$,

and phosphorous oxychloride $(10 \mathrm{ml})$ were refluxed for 24 hours. After completion of the reaction,(monitored by TLC), the reaction flask contents were kept to cool at $25{ }^{\circ} \mathrm{C}$, then carefully poured into a crushed-ice bath and washed with sodium bicarbonate solution $(10 \%)$. The solid was separated off and recrystallized from dimethylsulfoxide

[13].

Synthesis of 7,7'-(1,4-phenylene) bis [(5substitutedphenyl-1,3,4-oxadiazol-2-yl)-amino (7,8-dihydropyrazino[2,3-e][1,3]oxazepine-5,9dione)], compounds 3a-d:

Compound $2(0.001 \mathrm{~mol})$ and furo[3,4-b]pyrazine5,7-dione $(0.002 \mathrm{~mol})$ were dissolved in THF (10 $\mathrm{mL}$ ) and refluxed for 5 hours. A crystalline solid is filtered out, dried and recrystallized from ethanol after cooling in ice water bath [8].

Synthesis of 7,7'-(1,4-phenylene) bis [(5substitutedphenyl-1,3,4-oxadiazol-2-yl)-amino (7,8-dihydro-5 $H$-pyrazino[2,3-e $][1,3]$ diazepine5,9(6H)-dione)], compounds 4a-d:

The same procedure that employed to prepare 3a-d derivatives, was applied to synthesize compounds 4a-d via using $5 H$ pyrrolo[3,4- $b]$ pyrazine-5,7(6H)dione place of furo[3,4-b]pyrazine-5,7-dione.

Table 1. Physiochemical and microanalysis data of the synthesized compounds

\begin{tabular}{|c|c|c|c|c|c|c|}
\hline \multirow[b]{2}{*}{$\begin{array}{l}\text { Comp. } \\
\text { No. }\end{array}$} & \multirow[b]{2}{*}{$\mathrm{R}$} & \multirow[b]{2}{*}{$\begin{array}{l}\text { Empirical } \\
\text { Formula }\end{array}$} & \multirow[b]{2}{*}{$\begin{array}{l}\text { Mol.wt. } \\
(\mathrm{g} / \mathrm{mol})\end{array}$} & \multirow[b]{2}{*}{$\begin{array}{l}\text { Yield } \\
(\%)\end{array}$} & \multirow[b]{2}{*}{$\begin{array}{l}\mathrm{mp} \\
\left({ }^{\circ} \mathrm{C}\right)\end{array}$} & \\
\hline & & & & & & \begin{tabular}{ccc}
\multicolumn{3}{l}{ Elemental microanalysis Found (Calculated) } \\
\multirow{3}{*}{$\begin{array}{cc}\text { Molecular ion } \\
\% \mathrm{H}\end{array}$} \\
& $m / z($ rel. $\%)$ & \\
\end{tabular} \\
\hline 1 & & $\mathrm{C}_{10} \mathrm{H}_{14} \mathrm{~N}_{8} \mathrm{O}_{2}$ & 278.27 & 88 & $\begin{array}{c}138- \\
139\end{array}$ & C, 43.19 (43.16); H, $5.12(5,07) ; \mathrm{N}, 40.31$ (40.27) \\
\hline $2 \mathrm{a}$ & $\mathrm{CH}_{3}$ & $\mathrm{C}_{26} \mathrm{H}_{22} \mathrm{~N}_{8} \mathrm{O}_{2}$ & 478.51 & 69 & $\begin{array}{l}165- \\
166\end{array}$ & $\begin{array}{c}\mathrm{C}, 65.21(65.26) ; \mathrm{H}, 4.61(4.63) ; \mathrm{N}, 23.40(23.42) \\
479(15)\end{array}$ \\
\hline $2 b$ & $\mathrm{OCH}_{3}$ & $\mathrm{C}_{26} \mathrm{H}_{22} \mathrm{~N}_{8} \mathrm{O}_{4}$ & 510.50 & 77 & $\begin{array}{c}178- \\
179\end{array}$ & C, 61.11 (61.17); H, 4.33 (4.34); N, 21.89 (21.95) \\
\hline $2 \mathrm{c}$ & $\mathrm{NO}_{2}$ & $\mathrm{C}_{24} \mathrm{H}_{16} \mathrm{~N}_{10} \mathrm{O}_{6}$ & 540.45 & 81 & $\begin{array}{c}191- \\
193\end{array}$ & $\begin{array}{c}\mathrm{C}, 53.30 \text { (53.34); H, } 2.95(2.98) ; \mathrm{N}, 25.96(25.92) \\
541(10)\end{array}$ \\
\hline $2 d$ & $\mathrm{Br}$ & $\mathrm{C}_{24} \mathrm{H}_{16} \mathrm{Br}_{2} \mathrm{~N}_{8} \mathrm{O}_{2}$ & 608.24 & 76 & $\begin{array}{c}171- \\
173 \\
\end{array}$ & C, 47.35 (47.39); H, 2.64 (2.65); N, 18.39 (18.42) \\
\hline $3 \mathrm{a}$ & $\mathrm{CH}_{3}$ & $\mathrm{C}_{38} \mathrm{H}_{26} \mathrm{~N}_{12} \mathrm{O}_{8}$ & 778.69 & 72 & $\begin{array}{l}200- \\
202\end{array}$ & $\begin{array}{c}\mathrm{C}, 58.66(58.61) ; \mathrm{H}, 3.39(3.37) ; \mathrm{N}, 21.62(21.59) \\
780(07)\end{array}$ \\
\hline $3 b$ & $\mathrm{OCH}_{3}$ & $\mathrm{C}_{38} \mathrm{H}_{26} \mathrm{~N}_{12} \mathrm{O}_{10}$ & 810.69 & 76 & $\begin{array}{l}224- \\
227\end{array}$ & C, 56.28 (56.30); H, 3.21 (3.23); N, 20.71 (20.73) \\
\hline $3 \mathrm{c}$ & $\mathrm{NO}_{2}$ & $\mathrm{C}_{36} \mathrm{H}_{20} \mathrm{~N}_{14} \mathrm{O}_{12}$ & 840.63 & 68 & $\begin{array}{l}238- \\
239\end{array}$ & $\begin{array}{c}\mathrm{C}, 51.49 \text { (51.44); H, } 2.42(2.40) ; \mathrm{N}, 23.37 \text { (23.33) } \\
841(20)\end{array}$ \\
\hline $3 d$ & $\mathrm{Br}$ & $\mathrm{C}_{36} \mathrm{H}_{20} \mathrm{Br}_{2} \mathrm{~N}_{12} \mathrm{O}_{8}$ & 908.43 & 71 & $\begin{array}{l}221- \\
224\end{array}$ & C, 47.57 (47.60); H, 2.21 (2.22); N, 18.47 (18.50) \\
\hline $4 a$ & $\mathrm{CH}_{3}$ & $\mathrm{C}_{38} \mathrm{H}_{28} \mathrm{~N}_{14} \mathrm{O}_{6}$ & 776.72 & 81 & $\begin{array}{l}202- \\
204\end{array}$ & C, 58.81 (58.76); H, 3.65 (3.63); N, 25.29 (25.25) \\
\hline $4 \mathrm{~b}$ & $\mathrm{OCH}_{3}$ & $\mathrm{C}_{38} \mathrm{H}_{28} \mathrm{~N}_{14} \mathrm{O}_{8}$ & 808.72 & 87 & $\begin{array}{l}218- \\
221\end{array}$ & $\begin{array}{c}\mathrm{C}, 56.49(56.44) ; \mathrm{H}, 3.52(3.49) ; \mathrm{N}, 25.01 \text { (24.25) } \\
809(10)\end{array}$ \\
\hline $4 \mathrm{c}$ & $\mathrm{NO}_{2}$ & $\mathrm{C}_{36} \mathrm{H}_{22} \mathrm{~N}_{16} \mathrm{O}_{10}$ & 838.66 & 73 & $\begin{array}{l}234- \\
235\end{array}$ & C, 51.51 (51.56); H, 2.62 (2.64); N, 26.69 (26.72) \\
\hline $4 d$ & $\mathrm{Br}$ & $\mathrm{C}_{36} \mathrm{H}_{22} \mathrm{Br}_{2} \mathrm{~N}_{14} \mathrm{O}_{6}$ & 906.46 & 69 & $\begin{array}{l}226- \\
229\end{array}$ & $\begin{array}{c}\mathrm{C}, 47.74(47.70) ; \mathrm{H}, 2.46(2.45) ; \mathrm{N}, 21.68(21.63) \\
904(08)\end{array}$ \\
\hline
\end{tabular}




\section{Results and Discussion Chemistry}

In general, the synthetic strategy for the our compounds entitled 7,7'-(1,4-phenylene) bis[(5-substitutedphenyl-1,3,4-oxadiazol-2-yl)amino(7,8-dihydropyrazino[2,3-e][1,3]oxazepine-5,9dione)] 3a-d and 7,7'-(1,4-phenylene)bis[(5substitutedphenyl-1,3,4-oxadiazol-2-yl)-amino(7,8dihydro-5H-pyrazino[2,3-e][1,3]diazepine-5,9(6H)dione)] 4a-d is outlined in Scheme 1. At the beginning, the condensation reaction of starting materials 1,4-phenyldialdehyde (terephthaldehyde) and carbohydrazide was achieved in absolute ethanol and glacial acetic acid as solvent and catalyst, respectively. After completed the reaction, compound 1 was obtained and further cyclized with different substituents of 4-substituted benzoic acid in the presence of phosphorous oxychloride to give some new derivatives of bis 1,3,4-oxadiazole, 2a-d. The cyclization mechanism steps of 1,3,4-oxadiazoles 2ad synthesis are illustrated in Scheme 2 [14]. Whereas the cycloaddition reaction of $\mathbf{2} \mathbf{a}-\mathbf{d}$ in (THF) in dry conditions afforded new derivatives of pyrazino[2,3e][1,3] oxazepine-5,9-dione 3a-d and pyrazino[2,3e][1,3]diazepine-5,9(6H)-dione 4a-d, respectively. The reaction initially by attack of lone-pair of the imino group to the more electrophilic anhydride or imide carbonyl carbon, an active intermediate was produced which is easily closed by dipolar cycloaddition to form a seven-membered ring as a final product. The general reaction and mechanism steps are depicted in Scheme 3.

\section{Spectroscopic Analysis}

The structural assignments of the designed compounds have been deduced initially from their physical properties identification as well as the spectroscopic analysis. The most characteristic FT-IR absorption frequencies, ${ }^{1} \mathrm{H}$ NMR and ${ }^{13} \mathrm{C}$ NMR chemical shifts of the designed compounds are given in Table 2 with a good correspondence of the proposed structures. For example, FT-IR spectrum of compound 1 displayed medium bands at 3452-3217 $\mathrm{cm}^{-1}$ due to the stretching vibration of both $\mathrm{NH}_{2}$ and $\mathrm{NH}$ groups beside the significant peak of the imin group $\mathrm{C}=\mathrm{N}$ stretching in the region $1624 \mathrm{~cm}^{-1}$, Figure 1. On the other hand, FT-IR spectra of $\mathbf{2 a - d}$ revealed the disappearance stretching vibrational frequencies of the primary amino group. While the new bands of C-O-C of oxadiazole ring group were recorded at $1080-1245 \mathrm{~cm}^{-1}$ to an evidence of the reaction succeeding. Moreover, ${ }^{1} \mathrm{H}$ NMR spectrum of 1 Fig. 2, showed the most characteristic peak as a singlet at $6.23 \mathrm{ppm}$ caused by benzyliden imine proton (Ar$\mathrm{CH}=\mathrm{N}$ ) beside the other singlet peaks at 5.85 and $3.21 \mathrm{ppm}$ due to the primary amine protons $\left(\mathrm{NH}_{2}\right)$ and amidic proton (CO-NH), respectively [15]. In contrast, the primary amine protons peak was disappeared in the ${ }^{1} \mathrm{H}$ NMR spectrum of compounds 2a-d as shown in Fig 3.

As for compounds 3a-d and 4a-d, the FT-IR spectra indicated that the more distinguished strong and sharp bands in the region 1695-1631 $\mathrm{cm}^{-1}$ corresponded to the lactone and lactam $\mathrm{C}=\mathrm{O}$ groups stretching vibration, (see figure 4 ), beside the ${ }^{1} \mathrm{H}$ NMR spectra analysis Figs. 5 and 6. Furthermore, ${ }^{13} \mathrm{C}$ NMR spectra displayed other more significant signals at 163.6 and 164.3 ppm belonging to the $\mathrm{N}-\mathrm{C}=\mathrm{O}$ and $\mathrm{O}-\mathrm{C}=\mathrm{O}$, respectively in the 7-membered heteroaromatic systems, Fig. 7 [15]. In addition, the mass spectrum of compound $\mathbf{4 b}$ showed several peaks of main fragments with an agreement of the proposed structure and molecular formula, Fig 8. All elemental microanalyses and the spectroscopic data are given in Tables 1 and 2 .

\section{Bioactivity Investigation}

Antimicrobial activities of the titled compounds $3 \mathrm{a}-\mathrm{d}$ and $4 \mathrm{a}-\mathrm{d}$ were evaluated against various bacterial strains such as Staphylococcus Sciuri and Bacillus sphaericus (as gram positive), Eschrichia coli and Pseudomonas aeruginosa (as gram negative) beside the two fungal strains, Aspergillus flavus and Candida albicans by diffusion method [16]. DMSO was used to prepare $100 \mu \mathrm{g} / \mathrm{mL}$ concentrations of the examined compounds. For bacterial culture, plates have been incubated for 24 hours $/ 37^{\circ} \mathrm{C}$, whereas the fungal culture plates were kept at $25{ }^{\circ} \mathrm{C}$ and examined after 72 hours. Ciprofloxacin and fluconazole were used as reference standard drugs. The ability of growth inhibition for the tested compounds was determined as inhibition zone diameter and expressed in milliliters. The results indicated the good to excellent activities of designed compounds in comparison with standard references Table 3.

\section{Conclusion}

In summary, a series of new heterocyclic compounds of 7,7'-(1,4-phenylene) bis [(5-substitutedphenyl1,3,4-oxadiazol-2-yl)-amino (7,8dihydropyrazino[2,3-e][1,3] oxazepine-5,9-dione)] and 7,7'-(1,4-phenylene)bis [(5-substitutedphenyl1,3,4-oxadiazol-2-yl)-amino (7,8-dihydro-5 $\mathrm{H}$ pyrazino[2,3-e][1,3]diazepine-5,9(6H)-dione)] have been synthesized in good yields and cost effective procedures. A coincidence for the desired structures was achieved as deduced from their microanalysis and spectroscopy data. The target compounds have been investigated for their antimicrobial properties. Most of these new heterocycles exhibited good antibacterial and antifungal activities.

\section{Acknowledgement}

Authors are thankful to the Ministry of Science and Technology and to the University of AlMustansiriayah, Bagdad / Iraq for operating some of this work.

Table 2. Characteristic IR absorption frequencies, NMR chemical shifts of the designed compounds 


\begin{tabular}{|c|c|}
\hline No. & $\begin{array}{c}\text { Spectral data } \\
1 \mathrm{H} \mathrm{NMR}(400 \mathrm{MHz}, \mathrm{DMSO}-\mathrm{d} 6)(\delta, \mathrm{ppm}) / 13 \mathrm{C} \text { NMR }(100 \mathrm{MHz}, \mathrm{DMSO}-\mathrm{d} 6)(\delta, \mathrm{ppm})\end{array}$ \\
\hline 1 & $\begin{array}{l}\text { IR : 3452-3217 }\left(\mathrm{NH}_{2}, \mathrm{NH}\right), 3080\left(\mathrm{CH}_{\text {aro }}\right), 2982,2935\left(\mathrm{CH}_{\text {ali. }}\right), 1653(\text { Amide } \mathrm{C}=\mathrm{O}), 1624(\mathrm{C}=\mathrm{N}) . \\
{ }^{1} \mathrm{H} \text { NMR : } 7.61-7.28(\mathrm{~m}, 4 \mathrm{H}, \mathrm{ArH}), 6.23(\mathrm{~s}, 2 \mathrm{H}, \mathrm{Ar}-\mathrm{CH}=\mathrm{N}), 5.85(\mathrm{~s}, 4 \mathrm{H}, \mathrm{CO}-\mathrm{NH}-\mathrm{N}), 3.21\left(\mathrm{~s}, 4 \mathrm{H}, \mathrm{NH}_{2}\right) .\end{array}$ \\
\hline $2 a$ & $\begin{array}{l}\text { IR : 3410-3167 (NH), } 3080\left(\mathrm{CH}_{\text {aro }}\right), 2972,2899\left(\mathrm{CH}_{\text {ali. }}\right), 1660(\mathrm{C}=\mathrm{N}) .1261,1080(\mathrm{C}-\mathrm{O}-\mathrm{C}) . \\
{ }^{1} \mathrm{H} \text { NMR: } 8.71(\mathrm{~s}, 2 \mathrm{H}, \mathrm{Ar}-\mathrm{CH}=\mathrm{N}), 8.30-7.59(\mathrm{~m}, 12 \mathrm{H}, \mathrm{ArH}), 5.78(\mathrm{~s}, 2 \mathrm{H}, \mathrm{Ar}-\mathrm{C}-\mathrm{NH}-\mathrm{N}), 1.98\left(\mathrm{~s}, 6 \mathrm{H}, \mathrm{Ar}-\mathrm{CH}_{3}\right) .\end{array}$ \\
\hline $2 b$ & $\begin{array}{l}\text { IR : } 3321-3167(\mathrm{NH}), 3076\left(\mathrm{CH}_{\text {aro }}\right), 2992,2895\left(\mathrm{CH}_{\text {ali }}\right), 1645(\mathrm{C}=\mathrm{N}) .1242,1080(\mathrm{C}-\mathrm{O}-\mathrm{C}) . \\
{ }^{1} \mathrm{H} \text { NMR : } 8.33(\mathrm{~s}, 2 \mathrm{H}, \mathrm{Ar}-\mathrm{CH}=\mathrm{N}), 8.00-7.13(\mathrm{~m}, 12 \mathrm{H}, \mathrm{ArH}), 6.55(\mathrm{~s}, 2 \mathrm{H}, \mathrm{Ar}-\mathrm{C}-\mathrm{NH}-\mathrm{N}), 2.50\left(\mathrm{~s}, 6 \mathrm{H}, \mathrm{Ar}-\mathrm{OCH}_{3}\right) .\end{array}$ \\
\hline $2 c$ & $\begin{array}{l}\text { IR: } 3335-3193(\mathrm{NH}), 3071\left(\mathrm{CH}_{\text {aro }}\right), 1649(\mathrm{C}=\mathrm{N}) .1240,1076(\mathrm{C}-\mathrm{O}-\mathrm{C}) . \\
{ }^{1} \mathrm{H} \text { NMR: } 8.70(\mathrm{~s}, 2 \mathrm{H}, \mathrm{Ar}-\mathrm{CH}=\mathrm{N}), 6.98-7.59(\mathrm{~m}, 12 \mathrm{H}, \mathrm{ArH}), 4.78(\mathrm{~s}, 2 \mathrm{H}, \mathrm{Ar}-\mathrm{C}-\mathrm{NH}-\mathrm{N}) .\end{array}$ \\
\hline $2 d$ & $\begin{array}{l}\text { IR: } 3410-3170(\mathrm{NH}), 3070\left(\mathrm{CH}_{\text {aro }}\right), 1665(\mathrm{C}=\mathrm{N}) .1258,1080(\mathrm{C}-\mathrm{O}-\mathrm{C}) . \\
{ }^{1} \mathrm{H} \text { NMR: } 8.70(\mathrm{~s}, 2 \mathrm{H}, \mathrm{Ar}-\mathrm{CH}=\mathrm{N}), 6.98-7.59(\mathrm{~m}, 12 \mathrm{H}, \mathrm{ArH}), 4.78(\mathrm{~s}, 2 \mathrm{H}, \mathrm{Ar}-\mathrm{C}-\mathrm{NH}-\mathrm{N}) .\end{array}$ \\
\hline $3 a$ & $\begin{array}{l}\text { IR: } 3219-3149(\mathrm{NH}), 3074\left(\mathrm{CH}_{\text {aro. }}\right), 2958,2856\left(\mathrm{CH}_{\text {ali. }}\right), 1695(\text { Lactone } \mathrm{C}=\mathrm{O}), 1631(\mathrm{Lactam} \mathrm{C}=\mathrm{O}), 1614(\mathrm{C}=\mathrm{N}), 1244,1082(\mathrm{C}-\mathrm{O}-\mathrm{C}) \\
{ }^{1} \mathrm{H} \text { NMR: } 8.56(\mathrm{~s}, 4 \mathrm{H}, \mathrm{Pyrazine} \mathrm{CH}=\mathrm{N}), 8.33(\mathrm{~s}, 2 \mathrm{H}, \text { sec. amide } \mathrm{C}-\mathrm{CO}-\mathrm{N} \underline{\mathrm{H}}-\mathrm{C}), 7.87-7.02(\mathrm{~m}, 12 \mathrm{H}, \mathrm{ArH}), 6.62\left(\mathrm{~s}, 2 \mathrm{H}, \mathrm{Methine}-\mathrm{C}_{3} \mathrm{H}\right), \\
\text { 164.3,163.5 }(\mathrm{O}-\mathrm{C}=\mathrm{O}, \mathrm{N}-\mathrm{C}=\mathrm{O}) ; 147.9(\mathrm{~N}-\mathrm{C}=\mathrm{N}) ; 141.43-118.48(\text { Ar-Carbons}) ; 54.55(\mathrm{~N}-\mathrm{C}-\mathrm{N}) ; \\
\text { 43.38 }\left(\mathrm{CH}-\mathrm{CH}_{2}-\mathrm{C}=\mathrm{N}\right), 24.38(\mathrm{Ar}-\mathrm{CH} 3) \text {. }\end{array}$ \\
\hline $3 b$ & $\begin{array}{l}\text { IR: 3248-3128 (NH), 3081 }\left(\mathrm{CH}_{\text {aro. }}\right), 2951,2866\left(\mathrm{CH}_{\text {ali. }}\right), 1677(\text { Lactone } \mathrm{C}=\mathrm{O}), 1634(\mathrm{Lactam} \mathrm{C}=\mathrm{O}), 1627(\mathrm{C}=\mathrm{N}), 1240,1080(\mathrm{C}-\mathrm{O}-\mathrm{C}) \text {. } \\
{ }^{1} \mathrm{H} \text { NMR: } 9.02(\mathrm{~s}, 4 \mathrm{H}, \text { Pyrazine } \mathrm{CH}=\mathrm{N}), 8.41(\mathrm{~s}, 2 \mathrm{H} \text {, sec. amide C-CO-NH-C), 7.88-7.37 (m, 12H, ArH }), 6.82\left(\mathrm{~s}, 2 \mathrm{H}, \mathrm{Methine}-\mathrm{C}_{3} \mathrm{H}\right) \\
5.02(\mathrm{~s}, 2 \mathrm{H}, \mathrm{Ar}-\mathrm{C}-\mathrm{NH}-\mathrm{N}), 3.02\left(\mathrm{~s}, 6 \mathrm{H}, \mathrm{Ar}-\mathrm{OCH}_{3}\right) .\end{array}$ \\
\hline $3 c$ & $\begin{array}{l}\left.\text { IR: 3189-3140 (NH), } 3088\left(\mathrm{CH}_{\text {aro. }}\right), 2929,2853\left(\mathrm{CH}_{\text {ali. }}\right), 1669 \text { (Lactone } \mathrm{C}=\mathrm{O}\right), 1635(\text { Lactam } \mathrm{C}=\mathrm{O}), 1610(\mathrm{C}=\mathrm{N}), 1249,1078(\mathrm{C}-\mathrm{O}-\mathrm{C}) \\
{ }^{1} \mathrm{H} \text { NMR: } 9.28(\mathrm{~s}, 4 \mathrm{H}, \text { Pyrazine } \mathrm{CH}=\mathrm{N}), 8.13(\mathrm{~s}, 2 \mathrm{H}, \text { sec. amide C-CO-NH-C }), 7.94-7.37 \\
(\mathrm{~m}, 12 \mathrm{H}, \mathrm{ArH}), 6.44\left(\mathrm{~s}, 2 \mathrm{H}, \text { Methine }-\mathrm{C}_{3} \mathrm{H}\right) 5.21(\mathrm{~s}, 2 \mathrm{H}, \text { Ar-C-NH-N}) .\end{array}$ \\
\hline $3 d$ & $\begin{array}{l}\text { IR: } 3245-3146(\mathrm{NH}), 3068\left(\mathrm{CH}_{\text {aro. }}\right), 2955,2839\left(\mathrm{CH}_{\text {ali. }}\right), 1692(\text { Lactone } \mathrm{C}=\mathrm{O}), 1640(\mathrm{Lactam} \mathrm{C}=\mathrm{O}), 1624(\mathrm{C}=\mathrm{N}), 1244,1080(\mathrm{C}-\mathrm{O}-\mathrm{C}) \\
{ }^{1} \mathrm{H} \text { NMR: } 9.17(\mathrm{~s}, 4 \mathrm{H}, \text { Pyrazine } \mathrm{CH}=\mathrm{N}), 8.23(\mathrm{~s}, 2 \mathrm{H} \text {, sec. amide C-CO-NH-C), 7.68-7.08 (m, 12H, ArH }), 6.22\left(\mathrm{~s}, 2 \mathrm{H}, \mathrm{Methine}-\mathrm{C}_{3} \mathrm{H}\right) \\
\text { 4.81 (s, 2H, Ar-C-NH-N). }\end{array}$ \\
\hline $4 a$ & $\begin{array}{l}\text { IR: } 3241-3188(\mathrm{NH}), 3061\left(\mathrm{CH}_{\text {aro }}\right), 2960,2889\left(\mathrm{CH}_{\text {ali. }}\right), 1648(\mathrm{Lactam} \mathrm{C}=\mathrm{O}), 1624(\mathrm{C}=\mathrm{N}) .1243,1082(\mathrm{C}-\mathrm{O}-\mathrm{C}) \\
{ }^{1} \mathrm{H} \text { NMR: } 9.17(\mathrm{~s}, 4 \mathrm{H}, \mathrm{Pyrazine} \mathrm{CH}=\mathrm{N}), 8.23(\mathrm{~s}, 2 \mathrm{H}, \mathrm{sec} . \text { amide C-CO-N } \underline{\mathrm{H}}-\mathrm{C}), 7.58-7.02 \\
(\mathrm{~m}, 12 \mathrm{H}, \mathrm{ArH}), 6.74\left(\mathrm{~s}, 2 \mathrm{H}, \text { Methine }-\mathrm{C}_{3} \mathrm{H}\right) 4.81(\mathrm{~s}, 2 \mathrm{H}, \mathrm{Ar}-\mathrm{C}-\mathrm{NH}-\mathrm{N}), 1.72\left(\mathrm{~s}, 6 \mathrm{H}, \mathrm{Ar}_{-} \mathrm{CH}_{3}\right) . \\
{ }^{13} \mathrm{C} \text { NMR; } 162.11(\mathrm{~N}-\mathrm{C}=\mathrm{O}) ; 148.86(\mathrm{~N}-\mathrm{C}=\mathrm{N}) ; 143.87-120.48(\mathrm{Ar}-\mathrm{Carbons}) ; 59.52(\mathrm{~N}-\mathrm{C}-\mathrm{N}) ; 47.11\left(\mathrm{CH}-\underline{\mathrm{CH}}{ }_{2}-\mathrm{C}=\mathrm{N}\right), 22.02(\mathrm{Ar}-\mathrm{CH} 3) .\end{array}$ \\
\hline $4 b$ & $\begin{array}{l}\text { IR: } 3189-3160(\mathrm{NH}), 3085\left(\mathrm{CH}_{\text {aro }}\right), 2955,2855\left(\mathrm{CH}_{\text {ali. }}\right), 1660(\mathrm{Lactam} \mathrm{C}=\mathrm{O}), 1618(\mathrm{C}=\mathrm{N}) .1252,1081(\mathrm{C}-\mathrm{O}-\mathrm{C}) . \\
{ }^{1} \mathrm{H} \text { NMR: } 8.55(\mathrm{~s}, 4 \mathrm{H}, \text { Pyrazine } \mathrm{CH}=\mathrm{N}), 8.22(\mathrm{~s}, 2 \mathrm{H}, \mathrm{sec} \text {. amide } \mathrm{C}-\mathrm{CO}-\mathrm{NH}-\mathrm{C}), 7.83-7.12 \\
(\mathrm{~m}, 12 \mathrm{H}, \mathrm{ArH}), 6.72\left(\mathrm{~s}, 2 \mathrm{H}, \text { Methine }-\mathrm{C}_{3} \mathrm{H}\right) 3.41(\mathrm{~s}, 2 \mathrm{H}, \mathrm{Ar}-\mathrm{C}-\mathrm{NH}-\mathrm{N}), 2.72\left(\mathrm{~s}, 6 \mathrm{H}, \mathrm{Ar}-\mathrm{OCH}_{3}\right) .\end{array}$ \\
\hline $4 c$ & $\begin{array}{l}\text { IR: } 3245-3180(\mathrm{NH}), 3088\left(\mathrm{CH}_{\text {aro }}\right), 2958,2889\left(\mathrm{CH}_{\text {ali. }}\right), 1645(\text { Lactam } \mathrm{C}=\mathrm{O}), 1610(\mathrm{C}=\mathrm{N}) .1243,1089(\mathrm{C}-\mathrm{O}-\mathrm{C}) \\
{ }^{1} \mathrm{H} \text { NMR: } 8.57(\mathrm{~s}, 4 \mathrm{H}, \text { Pyrazine } \mathrm{CH}=\mathrm{N}), 8.05(\mathrm{~s}, 2 \mathrm{H}, \mathrm{sec} \text {. amide } \mathrm{C}-\mathrm{CO}-\mathrm{NH}-\mathrm{C}), 7.88-7.31 \\
(\mathrm{~m}, 12 \mathrm{H}, \mathrm{ArH}), 6.64\left(\mathrm{~s}, 2 \mathrm{H}, \text { Methine }-\mathrm{C}_{3} \mathrm{H}\right) 4.81(\mathrm{~s}, 2 \mathrm{H}, \mathrm{Ar}-\mathrm{C}-\mathrm{NH}-\mathrm{N}) .\end{array}$ \\
\hline $4 d$ & $\begin{array}{l}\text { IR: } 3269-3193(\mathrm{NH}), 3080\left(\mathrm{CH}_{\text {aro }}\right), 2972,2879\left(\mathrm{CH}_{\text {ali. }}\right), 1665(\text { Lactam } \mathrm{C}=\mathrm{O}), 1637(\mathrm{C}=\mathrm{N}) .1250,1088(\mathrm{C}-\mathrm{O}-\mathrm{C}) \text {. } \\
{ }^{1} \mathrm{H} \text { NMR: } 9.38(\mathrm{~s}, 4 \mathrm{H}, \text { Pyrazine } \mathrm{CH}=\mathrm{N}), 8.77(\mathrm{~s}, 2 \mathrm{H}, \text { sec. amide } \mathrm{C}-\mathrm{CO}-\mathrm{NH}-\mathrm{C}), 7.98-7.42 \\
(\mathrm{~m}, 12 \mathrm{H}, \mathrm{ArH}), 6.92\left(\mathrm{~s}, 2 \mathrm{H}, \text { Methine }-\mathrm{C}_{3} \mathrm{H}\right) 5.81(\mathrm{~s}, 2 \mathrm{H}, \mathrm{Ar}-\mathrm{C}-\mathrm{NH}-\mathrm{N}) .\end{array}$ \\
\hline
\end{tabular}

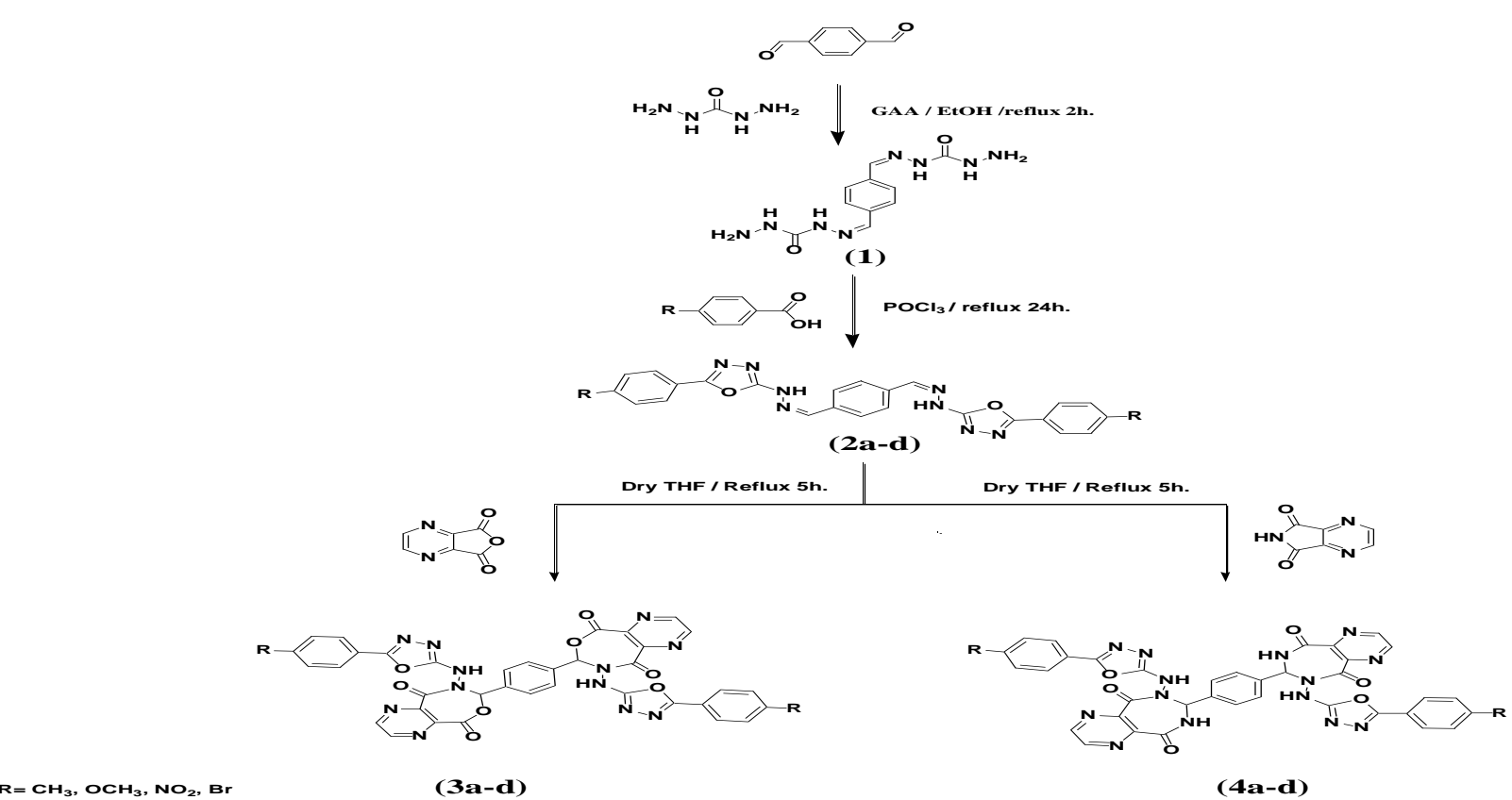

Scheme 1. General synthetic route for the designed compounds 


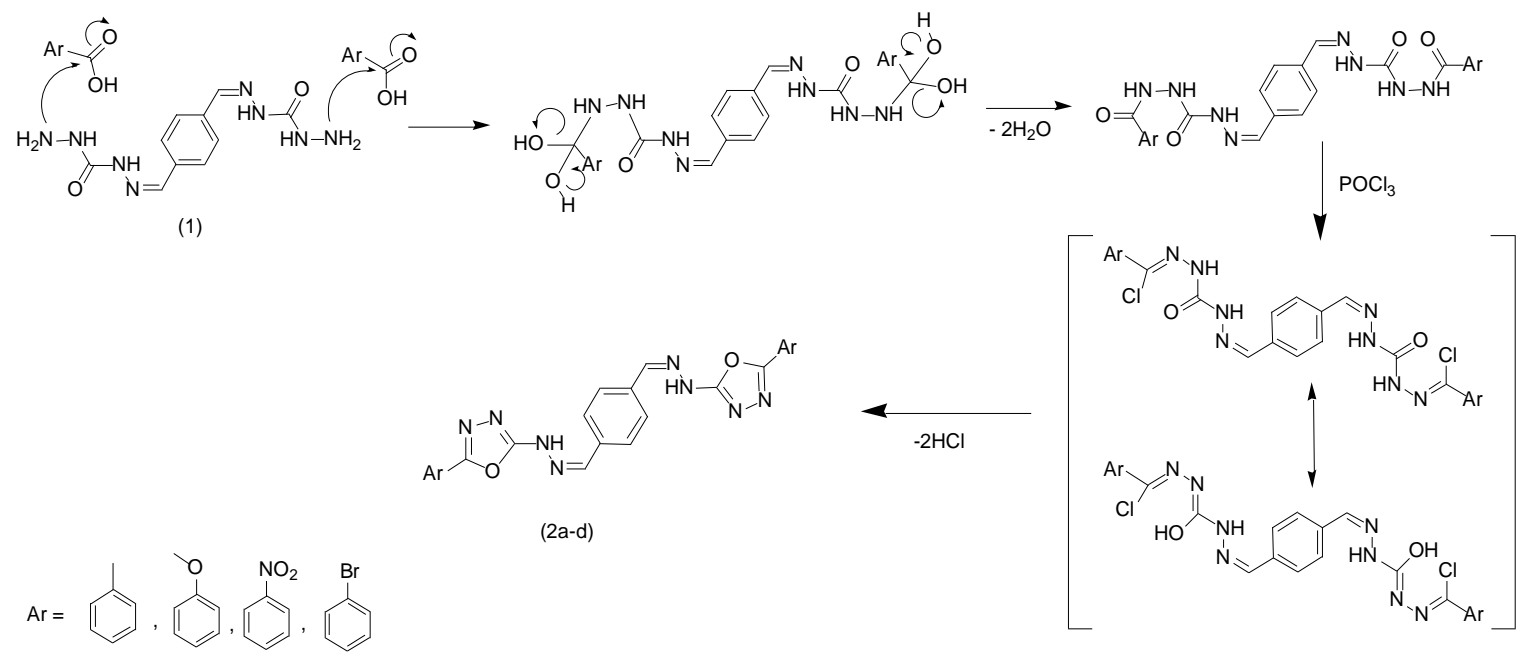

Scheme 2. General mechanism of the reaction synthesis of 2 a-d compounds
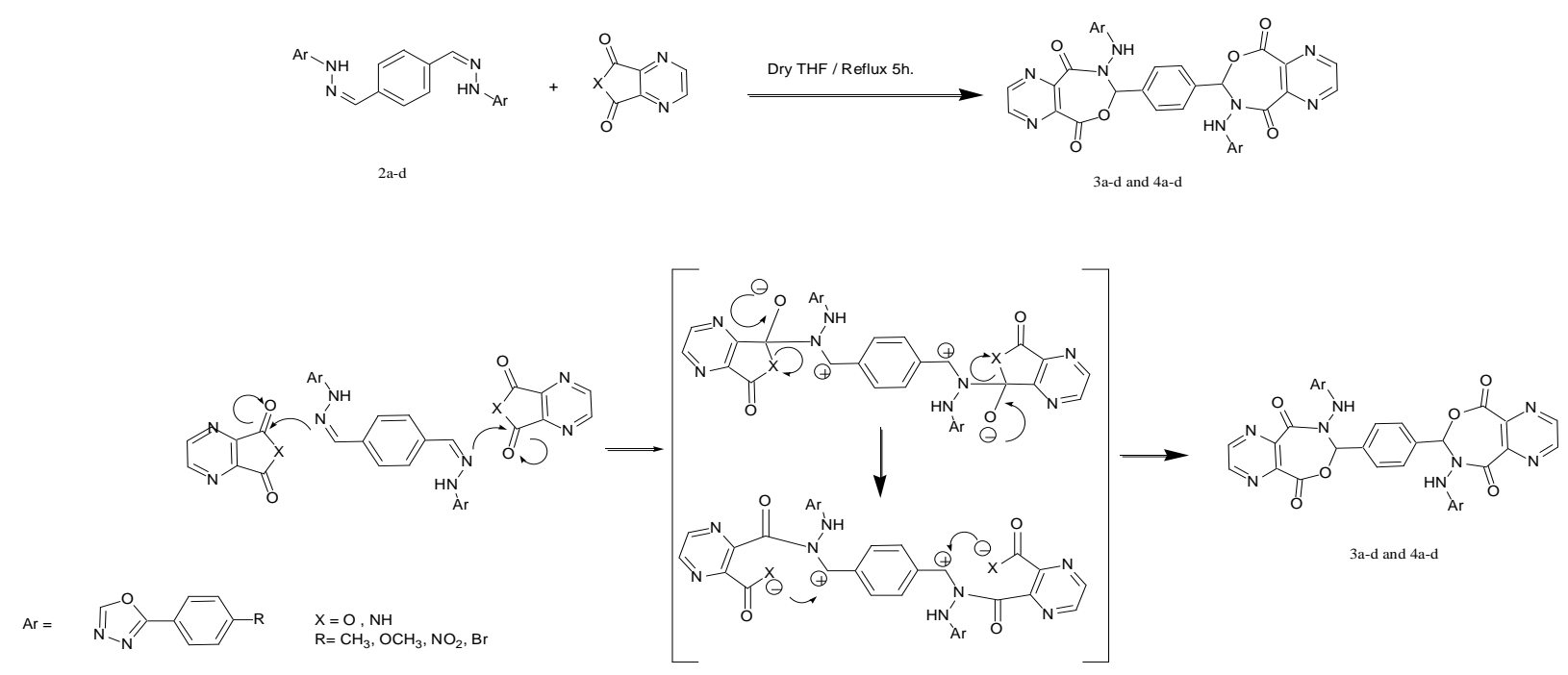

Scheme 3. General synthetic reaction and suggested mechanism of the target compounds 3a-d and 4a-d

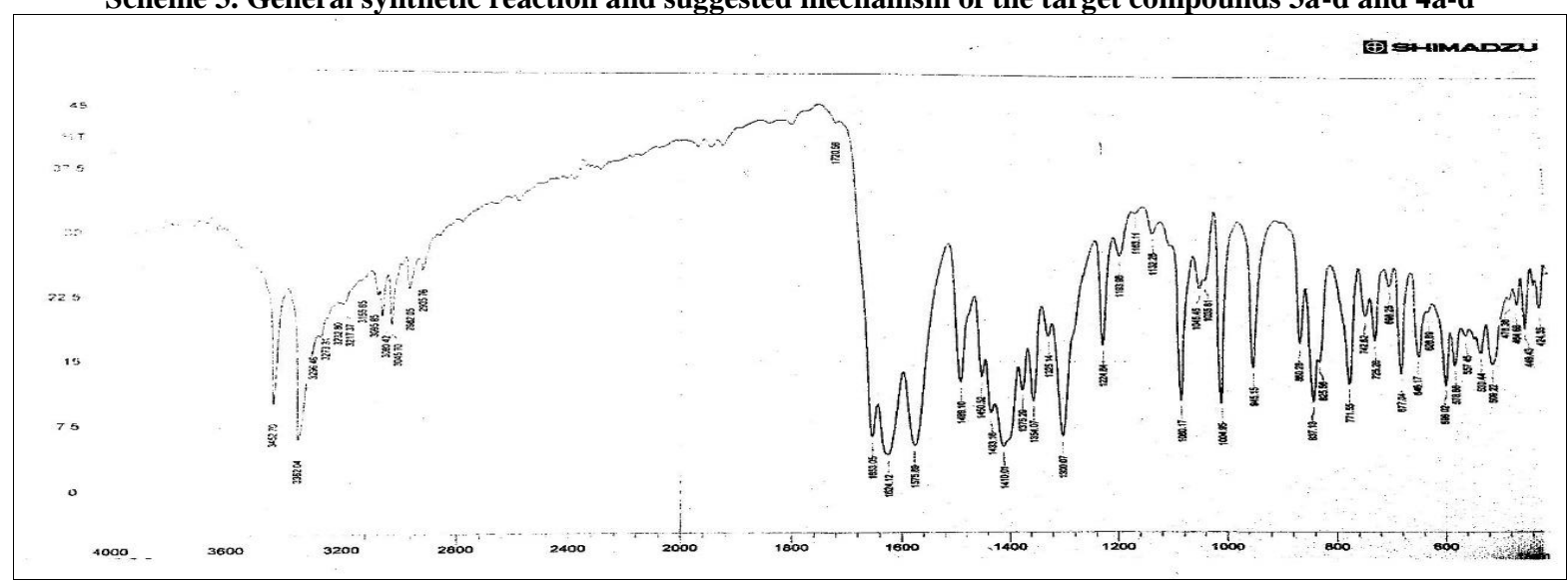

Fig 1. FT-IR spectrum of compound 1 
2950

A. N. Ayyash et al.

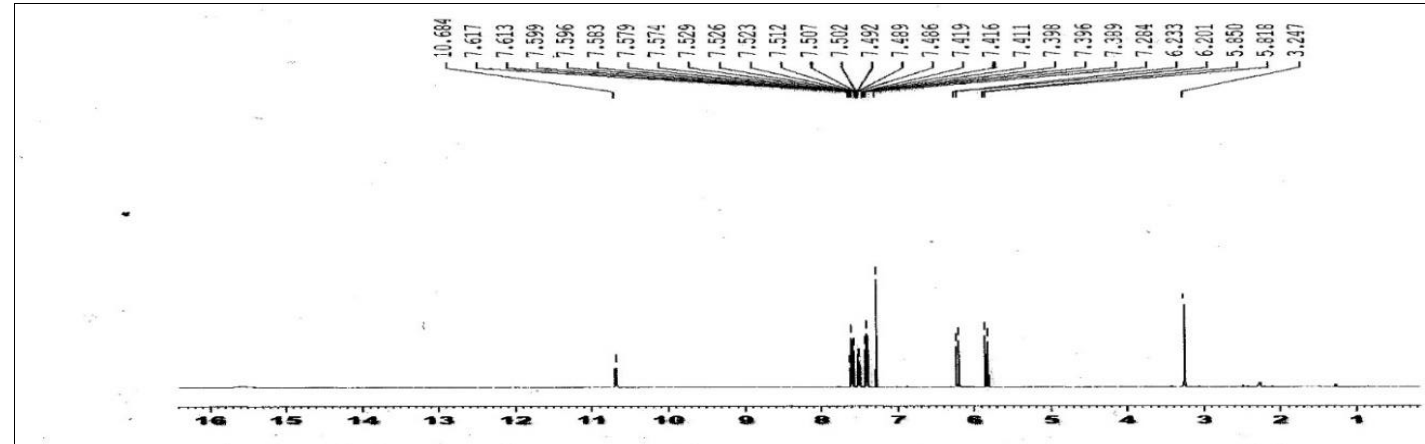

Fig 2. ${ }^{1} \mathrm{H}$ NMR spectrum of 1

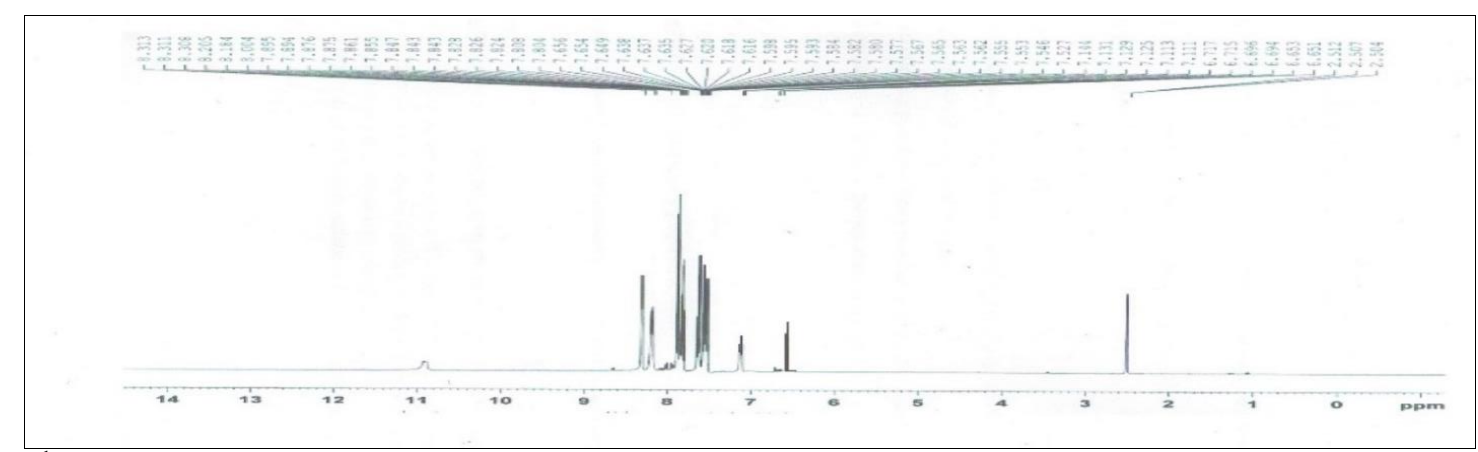

Fig 3. ${ }^{1} \mathrm{H}$ NMR spectrum of $2 \mathrm{~b}$

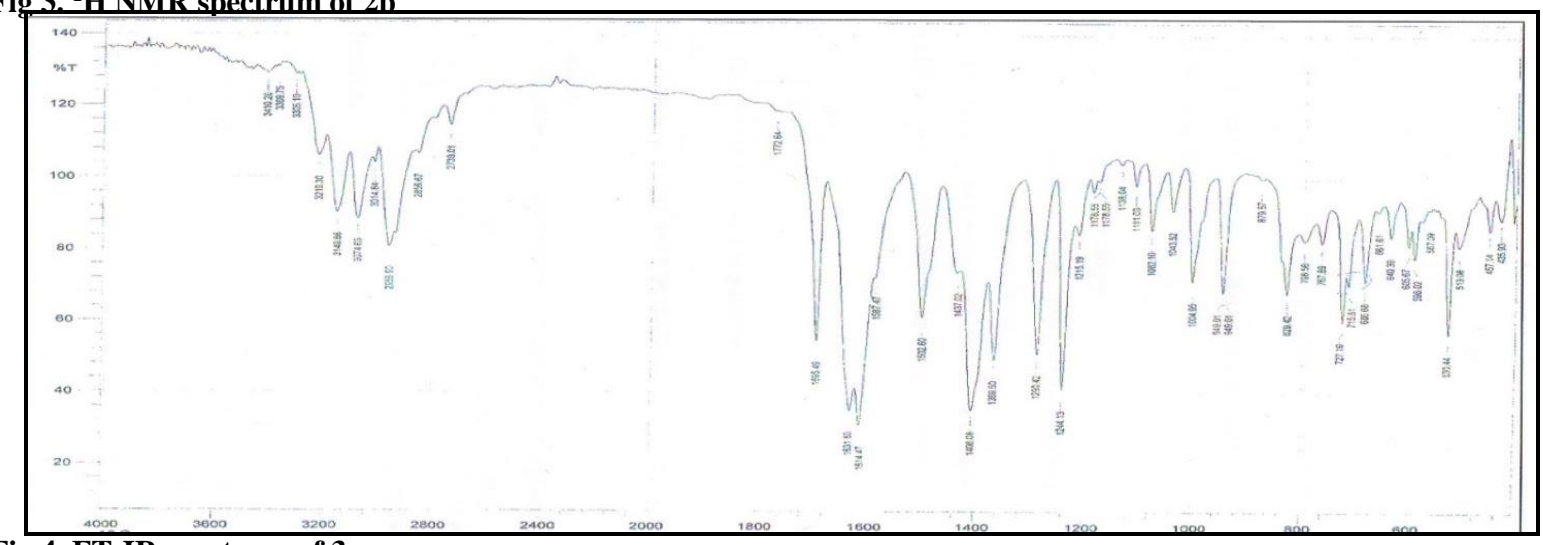

Fig 4. FT-IR spectrum of Bc

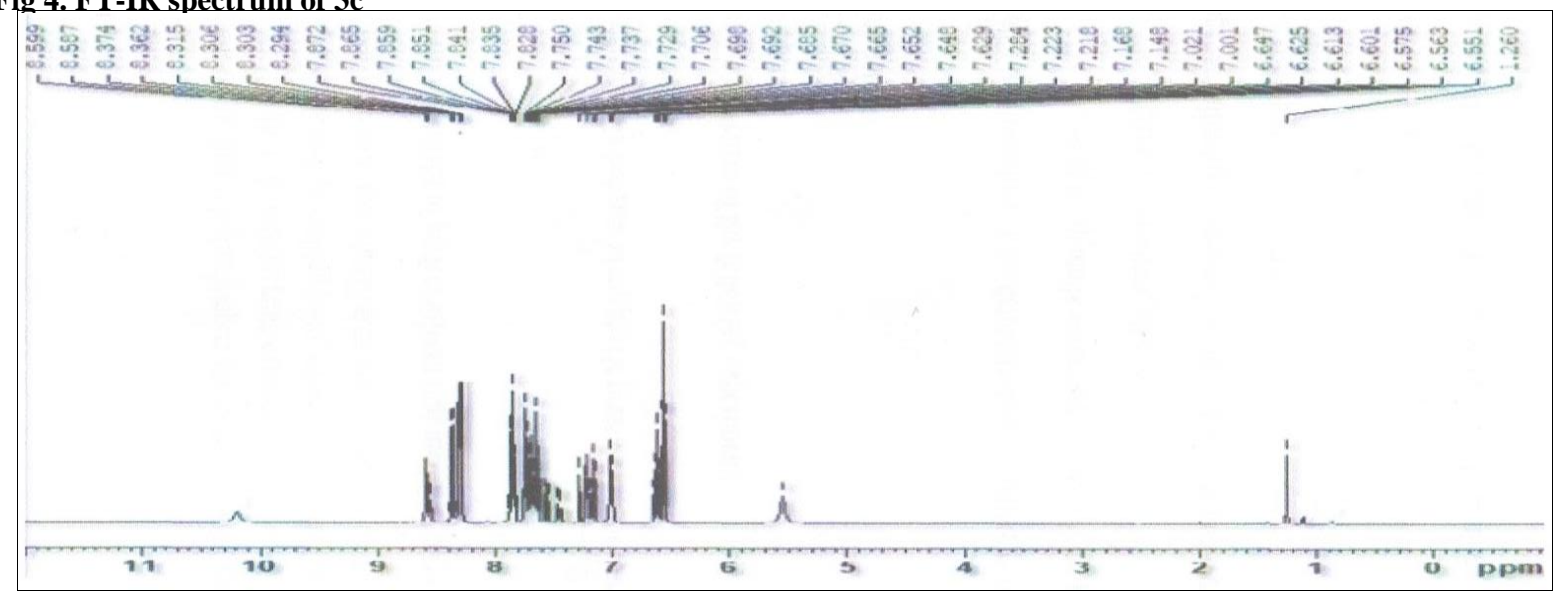

Fig 5. ${ }^{1} \mathrm{H}$ NMR spectrum of $3 a$

Egypt. J. Chem. 64, No. 6 (2021) 


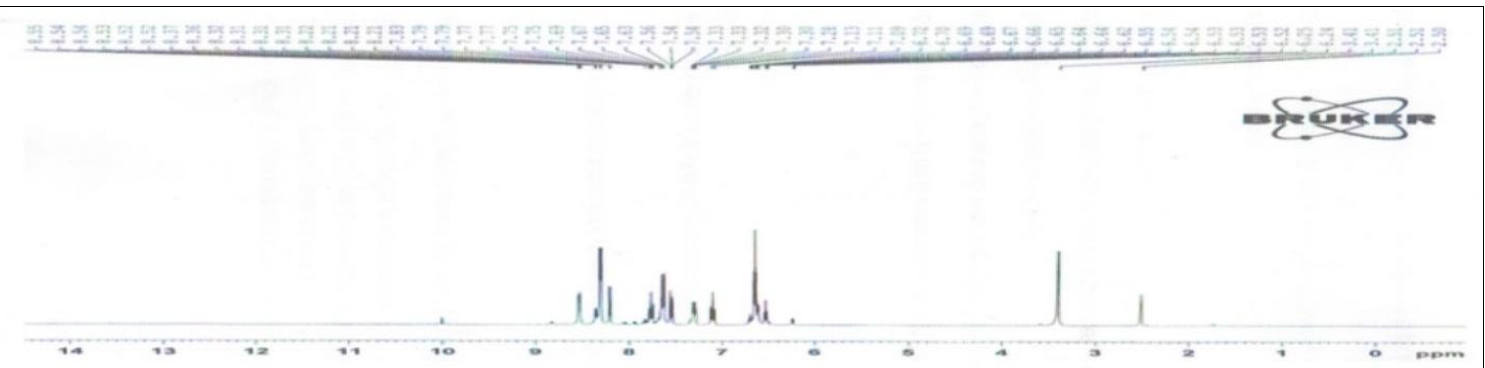

Fig 6. ${ }^{1} \mathrm{H}$ NMR spectrum of $4 \mathrm{a}$

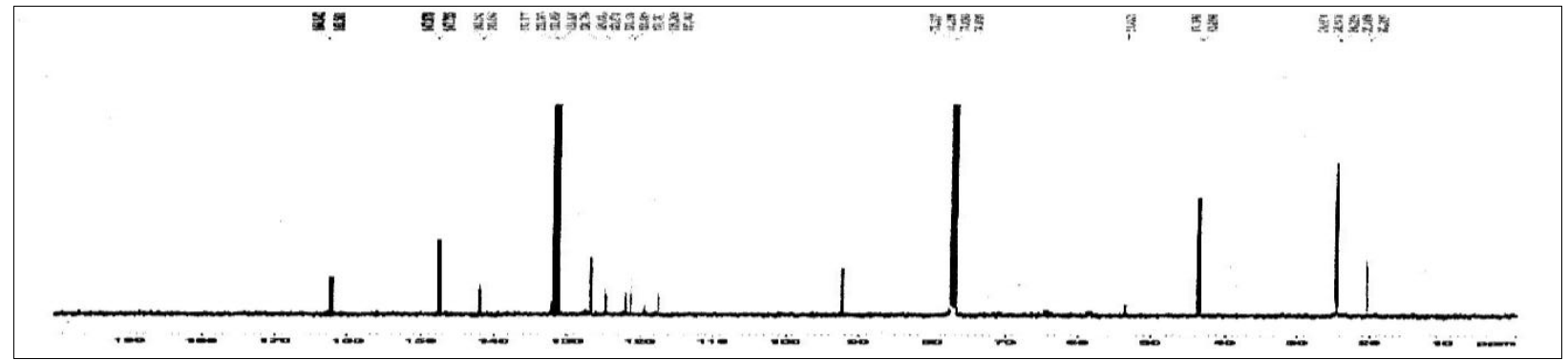

Fig 7. ${ }^{13} \mathrm{C}$ NMR spectrum of $3 \mathrm{a}$

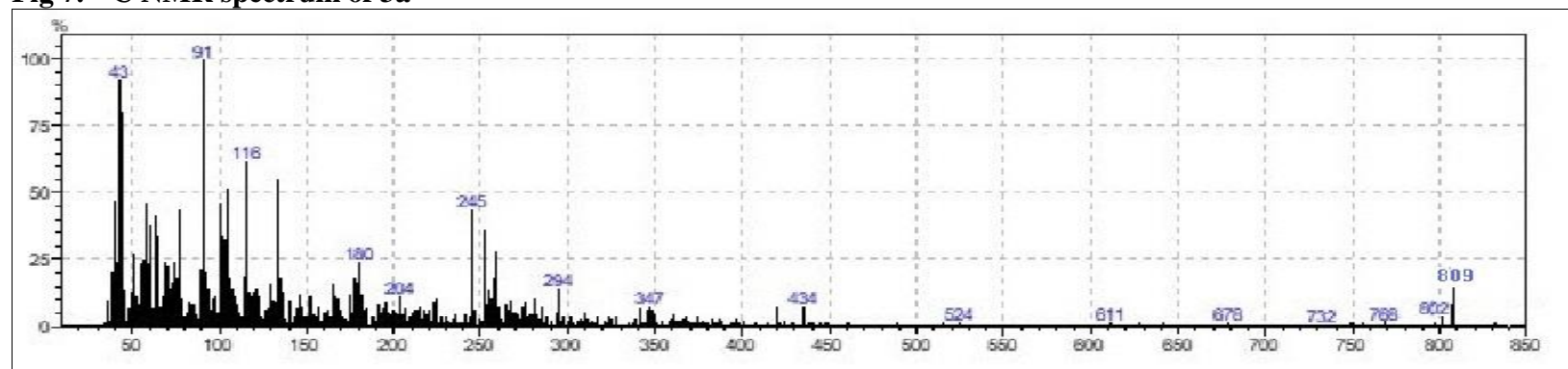

Fig 8. GC-MS spectrum of $4 \mathrm{~b}$

Table 3. Antimicrobial activities of newly synthesized compounds

\begin{tabular}{|c|c|c|c|c|c|c|}
\hline \multicolumn{7}{|c|}{ Zone of Inhibition (mm) } \\
\hline & \multicolumn{4}{|c|}{ Bacterial Strains } & \multicolumn{2}{|c|}{ Fungal Strains } \\
\hline & \multicolumn{3}{|c|}{ gram positive } & \multirow{3}{*}{$\frac{\text { Pseudomonas }}{\underline{\text { Aeruginosa }}}$} & \multirow{3}{*}{$\frac{\text { Aspergillus }}{\text { flavus }}$} & \multirow{3}{*}{$\begin{array}{l}\underline{\text { Candida }} \\
\underline{\text { albicans }}\end{array}$} \\
\hline Compd. & Staphylococcus & Bacillus & Eschrichia & & & \\
\hline No. & $\underline{\text { Sciuri }}$ & $\underline{\text { Sphaericus }}$ & $\underline{\text { Coli }}$ & & & \\
\hline $3 a$ & 14 & 15 & 16 & 18 & 19 & 24 \\
\hline $3 b$ & 14 & 16 & 16 & 15 & 17 & 18 \\
\hline $3 \mathrm{c}$ & 19 & 14 & 19 & 15 & 14 & 21 \\
\hline $3 d$ & 20 & 13 & 17 & 20 & 20 & 22 \\
\hline $4 \mathrm{a}$ & 15 & 11 & 15 & 14 & 18 & 19 \\
\hline $4 \mathrm{~b}$ & 12 & 14 & 18 & 12 & 18 & 21 \\
\hline $4 \mathrm{c}$ & 17 & 16 & 20 & 18 & 17 & 20 \\
\hline $4 \mathrm{~d}$ & 15 & 14 & 18 & 16 & 16 & 26 \\
\hline $\begin{array}{c}\text { Ciproflo } \\
\text { xacin }\end{array}$ & 16 & 16 & 22 & 22 & 18 & 19 \\
\hline $\begin{array}{c}\text { Flucona } \\
\text { zole }\end{array}$ & & & & & 20 & 24 \\
\hline
\end{tabular}

*concentration $(100 \mu \mathrm{g} / \mathrm{mL})$, diameter $(\mathrm{mm})$; milliliter 


\section{References}

[1] Lader M., Tylee A. and Donoghue J., Withdrawing benzodiazepines in primary care. CNS Drugs, 23 (1), 19-34 (2009).

[2] Plech T. et al., Synthesis and antimicrobial activity of thiosemicarbazides, s-triazoles and their Mannich bases bearing 3-chlorophenyl moiety. Eur. J. Med. Chem. 60, 128-134(2013).

[3] Gabriela L. et al., Synthesis, characterization and antibacterial activity of some triazole Mannich bases carrying diphenyl sulfone moieties. Eur. J. Med. Chem., 44, 3083-3087(2009).

[4] Aggarwal N. et al., Synthesis, antimicrobial evaluation and QSAR analysis of novel nalidixic acid based 1,2,4-triazole derivatives. Eur. J. Med. Chem. 46, 4089-4095(2011).

[5] Tariq S. and Pulisetty S., Pharmacotherapy for Insomnia. Clin. Geriatr. Med. 24, 1, 93-105(2008).

[6] Bodke D., Biradar S. and Kenchappa R., Synthesis, Characterization, and Biological Evaluation of 2-(2substituted)-5-phenyl-1,3,4-oxadiazole and 3,6 diphenyl[1,2,4]triazole[3,4-][1,3,4]thiadiazole Derivatives.

Indian Journal of Advances in Chemical Science. 4(3): 269-275(2016).

[7] El Ashry H. et al., A novel trans-amination process in 3-arylamino-5,5-dimethylcyclohex-2-en-1-one with nucleophiles and antimicrobial activity of selected products. Mediterranean Journal of Chemistry. 7(6): 452-462(2019).

[8] Ayyash A. and Abid O., Synthesis and Characterization of New Derivatives of $1 \mathrm{H}-2,3-$ Disubstituted-[1, 2-e] [1, 3]-Benzodiazepine-4, 7Dione. International Journal of Applied and Natural Sciences. 2(3): 69-74(2013).

[9] Kumarie N. et al., Synthesis and characterization of thiazepine derivatives and their biological activity. Indian J. Chem. Sec. B. 56, 1265-1269(2017).
[10] Abid O., Tawfeeq H. and Muslim R., Synthesis and Characterization of Novel 1,3-oxazepin-5(1H)-one Derivatives via Reaction of Imine Compounds with Isobenzofuran-1(3H)-one. Acta Pharm. Sci. 55 (4): 43 55(2017).

[11] Kakkerla R. et al., A Facile and simple synthesis of novel isoxazolylbenzo[f][1,4]oxazepine-3-(2H)-ones and their antimicrobial activity. Indian J. Chem. Sec. B. 57: 823-829(2018).

[12] Ayyash A., Tomma J., and Jaafer H., Synthesis and Antimicrobial Activity Evaluation of Some Novel Imides and Schiff Bases Containing 1,3,4Thiadiazoline Ring. International Journal of Applied and Natural Sciences. 3 (4): 61-66(2014).

[13] Tomi I. H., Al-Qaisi A. H., and Al-Qaisi Z. H., Synthesis, Characterization and Effect of Bis-1,3,4Oxadiazole Containing Glycine Moiety on the Activity of Some Transferase Enzymes. Ibn Al-Haitham Journal for Pure and Applied Science. 23 (3): 133141(2010).

[14] Nikpour F., Kazemi S. and Sheikh D., A Facile and Convenient Synthesis of $1 \mathrm{H}-\mathrm{Iso}$ indole-1,3(2H)diones. Heterocycles. 68(8): 1559-1564(2006).

[15] Silvrstein R.M., Welster and F.X. and Kienle D.J., Spectrometric Identification of Organic Compounds. John Wiely and Sons Inc., New York. (2005).

[16] Arthington-Skaggs B., Motley M. and Morrison C.J., Comparative evaluation of PASCO and national committee for clinical laboratory standards M27-A broth microdilution methods for antifungal drug susceptibility testing of yeasts. C. J. Clin. Microbiol. 38: 2254-2259(2000).

\section{تحضير مركبات جديدة فعالة بيولوجيا من مشتقات بايرازينو (e- 3،2، (2، (13) (13) اوكسازيبين و بايرازينو

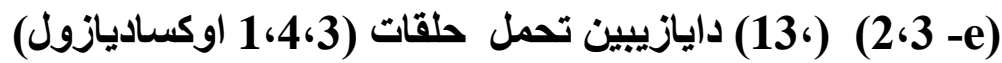

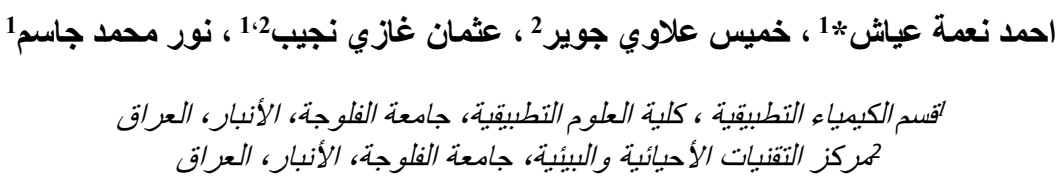

تم تحضير مجمو عـة جديدة من مشتقات الباير ازينو اوكسـازبين دايكاربوكسيليك انهيدريد او الباير ازين دايكاربوكسي ايميد مـع مشتقات جديدة من ونس

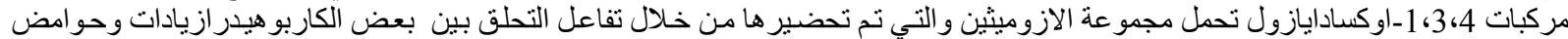
كربو كسيلية مختلفة بوجود البروتوني و الكربوني بالإضافة الى اطياف الكتلة. تم تسجيل وتحديد الفعالية الميكروبية للمركبات المحضرة وكانت النتائج جيدة إلى ممثازة. 